\title{
Diffusion treatment of quantum theory and gravity. 2. Diffusion gravity
}

\begin{abstract}
Zahid Zakir
Abstract

In the diffusion quantum mechanics (DQM) described in the first paper, the conservative diffusion of classical particles in a background field with a uniform energy density leads to the formalism of quantum mechanics. DQM provides a physical explanation for two fundamental facts - fluctuations in the energy of particles in the background field (their "thermal" energy) is manifested as their rest energy, and a corresponding decrease in the energy of the background field particle's vicinity appears as gravity. The influence of one particle on the background field is insignificant, but a very large number of particles in a small region noticeably reduces the local energy density of the background field. This reduces the local velocity of particle fluctuations, and also leads to the thermal diffusion flux of particles into this region. The increments of velocity, due to the conservativity of diffusion, cumulative and the appearing thermal diffusion acceleration does not depend on the masses of accelerated particles. As a result, the world lines of particles are curved identically and all processes with them slowdown, which means time dilation. Thus, the local energy deficit of the background field, generating conservative thermal diffusion, reproduces the basic properties of gravity. The effective metrics, connection and curvature appear on the hypersurface of simultaneity $\mathrm{t}=$ const., where the background field is defined. The Einstein's equations follow from the balance of energies in the system "the source + background field". Gravitation, as a result, appears as a consequence of the DQM, representing the manifestation of quantum fluctuations of particles in the inhomogeneous background field, i.e. as the diffusion gravity. Some observable effects of the diffusion gravity in astrophysics and cosmology are discussed.
\end{abstract}

Keywords: quantum fluctuations, vacuum energy, thermal diffusion, metrics, curvature

\section{Content}

Introduction . .2

1. Consequences of fluctuations of particles due to background field energy ..........................3

1.1. The fluctuation energy of a particle as its rest energy .................................................... 3

1.2. The decreasing of background field energy due to the rest energy ................................. 3

2. Gravity as thermal diffusion in the background field ..........................................................4

2.1. The main ideas of the diffusion treatment of gravity ....................................................

2.2. The principle of equivalence as a consequence of thermal diffusion gravity ...................5

2.3. Newtonian field as the simplest consequence of thermal diffusion ................................6

3. Effective geometry as a result of thermal diffusion ............................................................8

3.1. Thermal diffusion induced metric in the background field ..................................... 8

3.2. Einstein's equations from the balance of energies in the background field....................... 10

4. Physics on the hypersurface of simultaneity of the background field ..............................11

4.1. The theory of gravity on hypersurfaces of simultaneity ......................................... 11

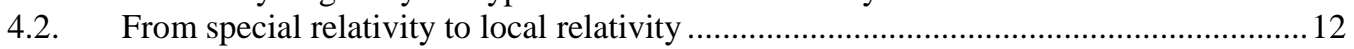

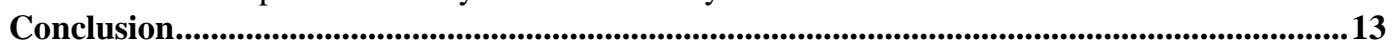

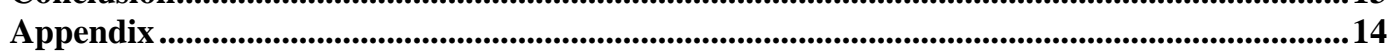

1. Conservative thermal diffusion of cold light gas in warm heavy ..................................... 14

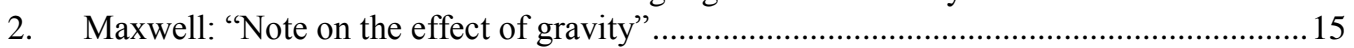

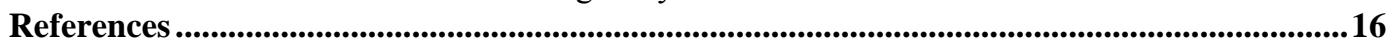

${ }^{1}$ Center for Theoretical Physics and Astrophysics, Tashkent Uzbekistan, zzakir@ qgph.org 


\section{Introduction}

In the first paper [1], diffusion quantum mechanics (DQM) was formulated as the theory of conservative (without friction) diffusion of classical particles in a fluctuating background field, in which the probability amplitudes are summed.

The formalism of quantum mechanics follows from the DQM in the particular case of diffusion in a homogeneous, or «isothermal», background field. Another consequence of DQM is the appearance of conservative thermal diffusion in the inhomogeneous background field. In the present paper the properties and consequences of this kind of thermal diffusion are studies.

In DQM, the total energy of the system "particle + background field" is conserved, and the average particle energy increases during quantum fluctuations due to a decrease in the background field energy to the same value. The influence on the background field of one or even many particles is extremely small, but a local decrease in the energy density $\rho_{V}$ of the background field generated by a very large concentration of particles perturbes the motion of particles in this region and in its vicinity.

Such a local decrease of $\rho_{V}$ reduces the intensity of fluctuations of the background field, which is equivalent to its effective "cooling". This, in turn, gives rise to a thermal diffusion flux of particles from regions with the usual $\rho_{V}$ to this region with a reduced $\rho_{V}$ and the concentration region of particles effectively attracts other particles.

Due to the conservativity of diffusion, the particle velocity increments accumulate and the velocity of flow increases with each shift. The resulting thermal diffusion acceleration of particles, as will be shown in the paper, depends only on the properties of the background field, but does not depend on the masses of accelerated particles. In the colder region, a decrease in the intensity of particle fluctuations also leads to thermal decelerations and contractions, i.e. proper times are slowing down and scales are shrinking.

These properties are characteristic for gravity and the fact that they follow from DQM at taking into account the influence of a high concentration of particles on the energy density of the background field shows that gravity can be interpreted as a manifestation of quantum phenomena under such specific conditions. Thus, DQM naturally leads to a thermal diffusion treatment of gravity, or diffusion gravity, reproducing the results of general relativity, but on the hypersurface of simultaneity, where the background field is defined.

It is shown in the paper that diffusion gravity leads to observable consequences in astrophysics and cosmology. For earlier versions of the new treatment, see [2], a systematical presentation will be given in the book [3]

In Section 1 the effects of particle's fluctuations in the background field and the inverse effect of particles on the background field itself are studied. In Section 2 the possibility of thermal diffusion treatment of gravity is considered. In Section 3 the inhomogeneity of the energy density of the background field leading to the effective metric and curvature of spacetime is studied. In Section 4 the main consequences of the diffusion gravity for particle physics and astrophysics are discussed. In the Appendix, examples of approximately conservative thermal diffusion are considered and Maxwell's comments of 1864 are presented in which the need for a medium with a large supply of positive energy to explain the nature of gravity is predicted [4]. 


\section{Consequences of fluctuations of particles due to background field energy}

\subsection{The fluctuation energy of a particle as its rest energy}

In the first paper [1], it was noted that the fluctuations of a classical particle in the background field lead to the transferring to this particle a "thermal" energy and this mean energy of fluctuations manifests itself as the rest energy, discovered earlier in the special theory of relativity. Here we consider the arguments leading to this conclusion.

The thermal energy of particles in an ordinary medium (gas, liquid) with temperature $T$ is equal to the average kinetic energy transferred to the particle by the molecules in collisions. It is proportional to the mass $m$ and rms velocity of the particles $v_{T}^{2}$. The same situation should be for particles fluctuating in the background field.

In DQM for a free particle $\rho=$ const. and it has no localization energy $U_{u}$ ("quantum potential"), and in the particle's rest frame ( $\mathbf{v}=0)$ there is no kinetic energy of drift. Therefore, the total energy of such a particle is reduced to the energy of (quantum) fluctuations $U_{m}$ :

$$
U_{m} \sim m v_{q}^{2}
$$

where $v_{q}$ is the «thermal» velocity of the particle's fluctuations in the background field.

In conservative diffusion, the "thermal" velocity $v_{q}$ does not depend on the properties of the diffusing particle, since according to DQM it should be the same in all inertial frames, and therefore equal to the light velocity $v_{q}=c$.

The main properties of such «thermal» energy of particles - proportionality to the mass of the particle and the square of the light velocity - are fully consistent with the properties of the rest energy $E_{0}=m c^{2}$, which is also known from the relativistic theory. Therefore, the energy of particle fluctuations $U_{m}$ can be identified with $E_{0}$, i.e. $\bar{U}_{m}=E_{0}$.

Thus, in DQM, the energy of fluctuations of particles of finite mass in the background field contains a constant part, the energy of "thermal" fluctuations, which in its rest frame manifests itself as rest energy:

$$
\bar{U}_{m}=m c^{2} .
$$

The presence of such a large energy accumulated by particles can be considered as indirect evidence of the existence of a background field as a source of this energy.

\subsection{The decreasing of background field energy due to the rest energy}

In DQM, the total energy of the system "particle + background field" is conserved, and part of the background field energy, equal to the rest energy of the particle, is expended on particle fluctuations. As a result, the energy of the background field itself in the vicinity of each particle becomes on average less to the value of the rest energy of the particle.

The effect of any particle on the background field is negligible, but at very large concentration of particles it leads to the new phenomena. Firstly, the arising inhomogeneity of the energy density of the background field affects the local intensity of fluctuations, as well as the particle drift velocity. Secondly, a decrease in the energy of the background field cannot be only where the particles are located, but appears as distributed around the neighborhood. There 
are questions about how this heterogeneity affects to the fluctuations and drift of particles, and according to what law is this local decrease in the energy density distributed in space?

In continuous media, when a cold compact accumulation of impurity particles is immersed in them, the particles of this cluster are sufficiently quickly heated to the temperature of the medium in this region, during which their average kinetic energy grows and tends to the thermal energy of the medium particles. This heating occurs due to local cooling of the medium with respect to other areas.

The result of local cooling of the medium, before its relaxation, is a decrease in the thermal energy of particles in this region, which reduces the intensity of fluctuations of all particles, both the medium and the cluster, in comparison with other regions with the same temperature. The appearance of a temperature gradient in the medium leads to a heat flux, as well as to a thermal diffusion flux of impurity particles.

In the case of a high concentration of classical particles in the background field, similar processes should occur. The intensity of particle fluctuations in this region should decrease due to a local decrease in the energy density of the background field, and this should lead to a thermal diffusion flux of other classical particles into this region with a lower intensity of fluctuations.

However, it is not obvious that the gradient of the energy density of the background field will necessarily lead to a "heat flux" in a more "cold" region and to the alignment of energy densities. The fact is that in this case the dilation of fluctuations means neither a little, nor a lot dilation of the local tempo of time, since here all processes are slowed down. The question of equalizing the rate of fluctuations at time dilation will be considered below.

\section{Gravity as thermal diffusion in the background field}

\subsection{The main ideas of the diffusion treatment of gravity}

In ordinary media, immersion of a compact cluster of a large number of "cold" impurity particles will lower the temperature of the medium in its vicinity and reduce the rate of fluctuations in this region. In approximately conservative thermal diffusion, impurity particles from other regions with faster fluctuations will drift into this region of slower fluctuations with an increasing drift velocity, and thermal diffusion acceleration occurs. In this case, the impurity particles are accelerated independently of each other, and therefore the acceleration of the cluster does not depend on the number of particles in the system, i.e. from the cluster's mass. Some examples of such thermal diffusion are presented in Appendix 1.

All this is similar to the properties of gravity, and therefore we can expect that gravity can be associated with the conservative thermal diffusion of classical particles in the background field with an inhomogeneous distribution of the energy density of this field.

The possibility of such a treatment of gravity in DQM naturally follows from the conservation of energy in the system "particle + background field", i.e. from the fact that during fluctuations of a particle, each random increase in its energy decreases the background field energy. The magnitude of the decrease in the average energy density of the background field at each point will then be the gravitational potential created by the particle at this point due to its quantum fluctuations.

However, this will be noticeable only at a very large concentration of particles, when their effect on the background field becomes significant. According to the DQM, part of the background field energy accumulated in the particle in the form of the energy of its fluctuations is equal to the particle's rest energy $\bar{U}_{m}=m c^{2}$ and therefore a massive body of $N$ particles lowers the background field energy to $N m c^{2}$. The energy of the gravitational field of this body 
can therefore be associated with a local deficit of the average energy of the background field due to the transition of this energy to the particles of the body as their rest energy.

The energy balance in the system "particle + background field" gives only a total change of the background field energy and in the general case does not determine its spatial distribution. In the case of a massive body, this density will depend on the relative arrangement of particles in it, which in the general case complicates the situation. However, in practice, symmetries can simplify the solution of the problem.

The described thermal diffusion treatment of gravity allows us to naturally explain the fact that gravity manifests itself as a change in the space-time geometry. In general relativity, this was postulated, while in the diffusion treatment of gravitation, as it will be shown below, of the effective metrics, connection and curvature of space-time follow from the behavior of world lines in a background field with an inhomogeneous energy density

\subsection{The principle of equivalence as a consequence of thermal diffusion gravity}

A full explanation of the physical nature of gravity should also include the relativistic effects. The diffusion treatment of gravity in DQM will therefore consist of a combination of quantum and relativistic phenomena. The difficulties in this way are obvious and therefore we will first consider, as a first step, the simplest cases - a weak and the constant in time field approximation.

This approximation is sufficient to deduce from the DQM those fundamental physical properties of gravity, from which the Newton's and Einstein's theories started. These consequences of DQM should manifest themselves even at the level of classical physics, i.e. after averaging over quantum fluctuations. Therefore, we will analyze the consequences of DQM in the framework of classical mechanics with relativistic corrections of order $1 / c^{2}$.

The energy of fluctuations of a particle, equal to its rest energy $E_{0}=m c^{2}$, represents that "energy level" occupied by a particle interacting with the background field. This energy level represents the energy of the «ground state» of the particle, from which it is natural to count the rest energies of particles near a massive body.

Particle's fluctuations near a massive body will be slower than at distant from it. This means that the fluctuation velocity near the body $c^{\prime}$ is less than the standard light velocity $c$. As a result, $c^{\prime}$ depends on the distance $r$ to the center of inertia of the body and $c^{\prime}(r) \leq c$.

The rest energy of the particle near the massive body, respectively, turns out to be equal to $E_{0}(r)=m c^{\prime 2}(r)$. This reduced rest energy can be expressed through the rest energy $E_{0}(\infty)=m c^{2}$ at large distance from the body, or the «ground state» energy, in the form:

$$
E_{0}(r)=m c^{\prime 2}(r)=m c^{2}+m \varphi(r) \geq 0 .
$$

Here $\varphi(r)$ is a difference in the mean square velocities of particle fluctuations in the background field near and at distance from the massive body:

$$
\varphi(r)=c^{\prime 2}(r)-c^{2} \leq 0, \quad \varphi(\infty)=0 .
$$

This function, as we see, plays the role of the "potential" of a particle in an effective external field, in the field of slowing down particle fluctuations in the vicinity of a massive body.

Thus, a change in the state of the background field near a massive body is reduced, in the first approximation, to the appearance of the potential energy $U(r)$ of particle's interaction 
with the effective external field proportional to the mass of the particle, and the potential of the field of slowing down of fluctuations $\varphi(r)$ :

$$
U(r)=m \varphi(r) \leq 0 .
$$

Moreover, the potential $\varphi(r)$, according to its definition (4), is negative-defined, although the value of the particle's «energy level» in the background field $E_{0}(r)$, according to (3), remains be positive-defined.

The Hamilton's function of the particle with the drift velocity $\mathbf{v}$ then takes the form:

$$
H=m c^{2}+m \varphi+\frac{\mathbf{p}^{2}}{2 m}, \quad \mathbf{p}=m \mathbf{v}
$$

The equation of motion following from (6) is known from Newtonian theory and has the form:

$$
\mathbf{a}=-\nabla \varphi,
$$

where $\mathbf{a}$ is the particle's acceleration.

As we see, the particle's mass falls out of the equation of motion (7) and all particles in the effective potential $\varphi(r)$ have the same acceleration regardless of their mass.

This consequence of the diffusion treatment of gravity expresses the main distinguishing property of gravity, which is not found in other fields. The principle of equivalence, formulated by Einstein on the basis of this property, led to an analogy with the behavior of particles in the accelerated frames of reference, and further, within the framework of the general relativity, it allowed to discover the geometric nature of gravity.

\subsection{Newtonian field as the simplest consequence of thermal diffusion}

The background field, which manifests itself through quantum fluctuations of classical particles, in the first approximation behaves like a scalar field $\phi$, since quantum fluctuations are the same in all inertial frames. At large distances from massive bodies, this field is, on average, uniform in space. But this field may be slightly dependent on time due to the cosmological expansion of space, which reduces the field energy density. This consequence of the new treatment will be discussed below, so here we will neglect such slow changes.

As discussed above, the homogeneity of the background field $\phi_{0}$ is violated near massive bodies, where the field potential is written in the form $\phi=\phi_{0}+\varphi, \phi_{0} \simeq$ const. If $\varphi$ were just a scalar field, then its density in the static case would be written in the form of a positive defined expression:

$$
H_{\varphi}=\frac{1}{2}(\nabla \varphi)^{2} \geq 0 \text {. }
$$

However, the diffusion treatment deals with the energy density of the background field near the massive body $H_{b f}(r)$, which can be written as the difference of the background field energy at large distance from the body $H_{b f}(\infty)$ and the energy density of the scalar field $H_{\varphi}$ (8), which is a deficit in the energy density of the background field near the body:

$$
H_{b f}(r)=H_{b f}(\infty)-H_{\varphi}=H_{b f}(\infty)-\frac{1}{2 B}(\nabla \varphi)^{2} .
$$


The constant addition $H_{b f}(\infty)$ does not affect under ordinary conditions, and the second term in (9), which is negatively defined, manifests itself as the energy of the gravitational field in the Newtonian approximation. From the correspondence with Newtonian theory, we then find the value of the constant $B=4 \pi G$, where $G$ is the gravitational constant, and as a result, the energy of the background field (9) takes the form:

$$
H_{b f}(r)=H_{b f}(\infty)-H_{\varphi}=H_{b f}(\infty)-\frac{(\nabla \varphi)^{2}}{8 \pi G} .
$$

Together with the particle's potential energy $m \varphi$, this expression gives for $\varphi$ the Poisson equation:

$$
\Delta \varphi=4 \pi G \rho,
$$

where $\rho$ is the mass density. The solution of this equation is the Newtonian potential:

$$
\varphi=-\frac{4 \pi G}{r} \int \rho d V
$$

Thus, thermal diffusion gravity in the first approximation leads to the Newtonian theory of gravity. Moreover, the negativity of the energy density of the Newtonian field is naturally explained, since this is not the energy density of an independently existing "gravitational" field, but is only a local deficit of the background field energy, determining the quantum fluctuations.

As early as 1864 , J. K. Maxwell noted that if the Newtonian gravitational potential is associated by the gravitational field, then the energy density of this field will be negative (as in (10)). Since he considered this unacceptable from the point of view of physics, he came to the conclusion that this fact allows us to say something about the yet unknown physical mechanism of gravity. Namely, he guessed that a reasonable way out of the situation can only be if there is a certain environment in the space with a huge supply of positive energy, which is larger in magnitude than the magnitude of the deepest of gravitational potential. Then the energy density of the gravitational field expresses as a local deficit in the energy density of this medium. The section "Note on the action of gravitational force" from the treatise "The dynamic theory of the electromagnetic field" [4], where Maxwell put forward this hypothesis, is given with abbreviations in Appendix 2. Here we give only his main conclusion:

"The assumption, therefore, that gravitation arises from the action of the surrounding medium in the way pointed out, leads to the conclusion that every part of this medium possesses, when undisturbed, an enormous intrinsic energy, and that the presence of dense bodies influences the medium so as to diminish this energy wherever there is a resultant attraction. As I am unable to understand in what way a medium can possess such properties, I cannot go any further in this direction in searching for the cause of gravitation"

As can be seen from the foregoing in this section, the problem of the negativity of the energy of the Newtonian field, which caused Maxwell's worry, is naturally solved in the diffusion gravity, and exactly as he suggested.

An estimation of how the rate of fluctuations decreases and how the local energy deficit is distributed in space, will be studied in more detail in the following sections. 


\section{Effective geometry as a result of thermal diffusion}

\subsection{Thermal diffusion induced metric in the background field}

The Lagrange function of the particle in the background field is found from the expression for $H$ in (6):

$$
L=\mathbf{p} \mathbf{v}-H=-m c^{2}-m \varphi+\frac{1}{2} m \mathbf{v}^{2} .
$$

The action function $S$ for the particle then takes the form:

$$
S=\int d t L=-m c \int d t\left(c+\frac{\varphi}{c}-\frac{1}{2} \frac{\mathbf{v}^{2}}{c}\right) .
$$

This action function can be written in a form analogous to the action function in the relativistic theory, expressed through the space-time interval $d s$ :

$$
S=-m c \int d s, \quad d s=d t\left(c+\frac{\varphi}{c}-\frac{1}{2} \frac{\mathbf{v}^{2}}{c}\right) .
$$

Squaring the expression for $d s$ in (15), and omitting the terms $\sim 1 / c^{2}$, we obtain:

$$
d s^{2} \simeq\left(1+\frac{2 \varphi}{c^{2}}\right) c^{2} d t^{2}-d \mathbf{r}^{2}=g_{00} c^{2} d t^{2}-d \mathbf{r}^{2} .
$$

From this we see that the appearance of the potential $\varphi(r)$ is equivalent to a change in the time component of the space-time metric, which becomes equal to:

$$
g_{00}=1+\frac{2 \varphi}{c^{2}} \text {. }
$$

From (16) it follows that, since $\varphi \leq 0$, the proper time interval of the standard clocks $d \tau$ resting near a massive body is less than the time interval $d t$ between the same events measured by the standard clocks at a distance:

$$
d \tau=d t \sqrt{g_{00}}=d t \sqrt{1+\frac{2 \varphi}{c^{2}}} .
$$

and we have $d \tau<d t$

The proper time of the distant clock $t$ here plays the role of "world" time, since its values for simultaneous events are the same everywhere. Thus, we are dealing with the dilation of the proper times of particles near a massive body with respect to the proper times of distant particles. This is a thermal diffusion dilation of proper times, which is nothing more than a gravitational time dilation.

As is known from general relativity, it follows from (18) that the conserved energy of a particle is the product of its energy $E_{0}$ to the time dilation factor $\sqrt{g_{00}}$ :

$$
E=E_{0} \sqrt{g_{00}} .
$$

Thermal equilibrium along such a body also satisfies the same condition:

$$
T=T_{0} \sqrt{g_{00}},
$$


where $T_{0}$ is the local temperature. The temperature $T$ is the same along the body and in the background field it corresponds to the conserved energy $E$ from (19). This explains the fact that local inhomogeneity in the background field does not become homogeneous as it does in ordinary media, where thermal conductivity and convection make the temperatures the same and then equilibrium is reached. In the background field, equilibrium occurs at two types of temperature, since the fluctuation velocities in world time remain the same, which leads to a new kind of "thermal equilibrium".

The local energy of particle fluctuations (3), i.e. their rest energy, therefore, becomes less and less as one approaches a massive body. From here follows one more consequence of the DQM - when the size of a massive spherical body is sufficiently small, at a certain value of the radius of its surface, almost all the energy of the background field in this region will become transferred to the energy of fluctuations of the particles of this body. As a result, in terms of the world time $t$ of a distant clock, the background field ceases to fluctuate test particles, i.e. the fluctuations of all particles in this region will freeze. A value of this radius $r_{g}$ follows from the vanishing condition for $g_{00}(r)$ :

$$
g_{00}\left(r_{g}\right)=1+\frac{2 \varphi\left(r_{g}\right)}{c^{2}}=0, \quad 2 \varphi\left(r_{g}\right)=-c^{2} .
$$

In general relativity, the basic properties of the gravitational field were postulated, and then they were presented as properties of the space-time geometry. Thus, the basic properties of gravity, such as the independence of acceleration from the masses of test particles and the energy-momentum of matter as a source of the field, follow from conservative thermal diffusion in the background field. To go to their geometrical form, it is enough to rely on standard methods of constructing the theory of gravity in the framework of general relativity, but on global hypersurfaces of simultaneity, where the background field is defined.

The independence of the thermal diffusion acceleration of test particles from their masses leads to the same acceleration of both particles and the basis of the local frames of reference. But the same acceleration of both objects and the local frames of reference is indistinguishable from the presence of a nontrivial metric and curvature of space-time.

During thermal diffusion in the background field, the average trajectories of free particles on the hypersurface of simultaneity, where the background field is defined, are not geodesics in flat space-time, but contain some deviations from geodesics. To describe them in relativistic kinematics, it is necessary to introduce curvilinear coordinates $x^{\mu}\left(x^{a}, t\right)$ and basis vectors $e_{\mu}^{a}$ along the mean trajectories of free particles, where their differentials are related to the local intervals of the physical coordinates of the test particle $d x^{a}$ at the point $M$ as $d x^{\mu}=e_{a}^{\mu} d x^{a}, \mu, v=0, \ldots, 3, a, b=0, \ldots, 3$.

Then thermal diffusion on the hypersurfaces $t=$ const . can be described as motion in an effective Riemannian manifold with the metric tensor $g_{\mu \nu}=e_{\mu}^{a} e_{v}^{a}$, where the mean trajectories of the thermal diffusion drift are geodesics. The theory of diffusion gravity, therefore, can be constructed as quantum mechanics in effective (pseudo) Riemannian spacetime on hypersurfaces $t=$ const. Therefore, one can use well-known methods for describing diffusion in curved manifolds. 
Thermal diffusion in the background field, therefore, induces a nontrivial effective metric $g_{\mu v}(x, t)$ determining the space-time interval between events:

$$
d s^{2}=\eta_{a b} d x^{a} d x^{b}=g_{\mu \nu} d x^{\mu} d x^{v} .
$$

For a test particle, the action function then takes the form:

$$
S=-m c \int d s=-m c \int \sqrt{g_{\mu \nu} d x^{\mu} d x^{v}} .
$$

The appearance of the effective metric allows one to introduce the thermal diffusion parallel transfer of tensors in flat space-time along the average trajectory of the drift:

$$
d e_{a}^{\mu}(x, t)=-\Gamma_{\nu \lambda}^{\mu} e_{a}^{\lambda} d x^{b}(t),
$$

where $\Gamma_{\nu \lambda}^{\mu}$ is the effective connectivity. The effective Riemann curvature tensor $R_{\mu \nu \lambda \sigma}$ corresponding to this connection is then introduced in the usual way.

Thus, the first postulate of general relativity, the principle of equivalence, as well as its direct consequence that gravity can be described as a non-trivial metric and space-time connection on the hypersurfaces $t=$ const. now appear as the consequences of thermal diffusion in the background field at a high concentration of matter.

\subsection{Einstein's equations from the balance of energies in the background field}

Let's turn to the second of the postulates of general relativity that gravity is generated by the energy-momentum of the source. In the diffusion treatment, the background field energy around the source is reduced by the fluctuation energy of its particles, including the rest energy and kinetic energy obtained from the background field during the formation of the source. In essence, in the diffusion treatment the total energy of the particle is equal to the energy to which the energy of the background field in its vicinity decreased.

Therefore, the sum of the total energy of the source's particles and the magnitude of the decrease in the background field energy in its vicinity is zero, which in terms of energy densities $\varepsilon_{m}$ for resting particles gives:

$$
\int\left(\rho_{v a c}^{(0)}-\rho_{v a c}\right) d V=\int \varepsilon_{m} d V
$$

The decrease in the energy density of the background field near the source and its smooth recovery with distance from it, appearing on the left side of (25), can be described both in terms of thermal diffusion and in terms of the gravitational potential or the corresponding curvature of space-time.

In a form more suitable for physical problems, the curvature tensor is used in the combined form as the Einstein tensor $G_{\mu \nu} \equiv R_{\mu \nu}-g_{\mu v} R / 2$. Choosing at each point a specific local basis with 4-velocity $u^{\mu}$ on a certain hypersurface of simultaneity and projecting tensors onto this basis, we obtain the corresponding scalars at each point.

In the diffusion treatment, the scalar projection $G_{\mu \nu}$ onto a hypersurface with a timelike unit normal $n^{\mu}$ can be identified with the energy-momentum deficit of the background field:

$$
\rho_{v a c}^{(0)}-\rho_{v a c}=\frac{1}{\kappa} G_{\mu \nu} n^{\mu} n^{v} .
$$


In the general case, the condition of the balance of energies of the source and the background field around it should be written locally and in the tensor form, i.e. through the energy-momentum tensor of the source $T_{\mu v}$, which leads to the Einstein equations:

$$
\frac{1}{\kappa} G_{\mu \nu}=T_{\mu \nu} .
$$

If it is possible to integrate energy scalars throughout the space on certain hypersurfaces of simultaneity, the total energies of the source and its gravitational field, in accordance with (25), should be equal to each other. As a consequence of a clearer physical picture, there are no problems with the energy of the gravitational field.

Thus, the theory of diffusion gravity is the next step in understanding the nature of gravitation after GR. From this theory, as it was shown above, both the principle of equivalence and the relationship of field quantities with the energy-momentum of matter follow naturally. Therefore, the new treatment reproduces the formalism of general relativity, but not as a formal mathematical model, but as a geometric method of describing thermal diffusion gravity without explanation its microscopic mechanism.

\section{Physics on the hypersurface of simultaneity of the background field}

\subsection{The theory of gravity on hypersurfaces of simultaneity}

The state of the background field, like any extended physical object, must be set globally, in the whole space at the same moment of time. For this, an extended frame of reference is needed, built as a set of simultaneously coexisting local frames of reference resting with respect to each other. This means that the background field must be set on the hypersurface of simultaneity of the "rigid" extended frame of reference.

From this it follows that the gravitational field, as the field of deformations of the background field, must also be defined on the hypersurfaces of simultaneity of the "rigid" extended frame of reference. This requirement for the theory must be satisfied even if its formalism allows one to go beyond these restrictions.

The latter refers to GR in describing processes in the gravitational field, which was the source of many misunderstandings and misconceptions. In general relativity, frames of reference with mutually moving local frames, such as comoving frames, were often introduced. The absence of a common hypersurface of simultaneity of the local elements of these frames of reference, as well as the impossibility of adequate physical treatment in such cases, were not seeming as problems.

In the theory of diffusion gravity, where gravity is a property of the background field, from a wide range of methods of description of gravitation, allowed by the general relativity formalism, only those are physically meaningful in which the above restriction is fulfilled. This is the describing of the field on the hypersurfaces of simultaneity as a necessary condition, since only at the simultaneous coexistence of parts of the field in different spatial points do they form such an extended object as a physical field.

This considerably changes the formulation of problems both in the theory of gravity and in its applications in particle physics, especially at Planck distances, as well as in astrophysics and cosmology. The physical picture arising from this approach may differ from that which followed from treatments based on coordinates and frames of reference, chosen arbitrarily for reasons of simplicity and convenience, rather than physical feasibility and admissibility. Some 
of the new effects revealed by this more realistic approach are discussed below, and others are considered in the third paper [3].

The apparent relativity of relativistic effects in the gravitational field led earlier to widespread misconceptions. In reality, local contraction of scales arises from the comparison of lengths on hypersurfaces of simultaneity, and time dilation - from the comparison of time intervals between hypersurfaces. These relativistic effects in the gravitational field, with the exception of kinematic effects, are absolute and observable by observers in any frames of reference or coordinates. This can be demonstrated in the case of two simple examples.

Let two pairs of simultaneous events at two points of the gravitational field $r_{1}$ and $r_{2}$ occur at two moments of world time. Suppose that at a zero moment of world time, standard clocks at these points also showed zero moments of proper time. At another moment of world time $t>0$ at the same points, we also consider two simultaneous events, when the standard clock at these points showed moments of proper time $\tau_{1}$ and $\tau_{2}$. The world time interval between two events at each point is the same and equal $t$. But the intervals of proper time that have elapsed between the same events at two points are not equal: $\tau_{1} \neq \tau_{2}$ and the interval of those standard clocks that were in a stronger gravitational field will be smaller, for example, if it's in $r_{1}$, then it will be $\tau_{1}<\tau_{2}$. Observers agree with this in any frame of reference, i.e. such a dilation is absolute.

Further, tangentially oriented standard scales in a spherical static field are not contracted and are the same as large distance from the source, while radially oriented scales are contracted. If on two close circles are placed $2 \pi r_{1}$ and $2 \pi r_{2}$ standard scales, then on the radial segment between them, due to scale contraction, not $\Delta r=r_{2}-r_{1}$ standard scales will fit, but more, and as more, as closer to the source of the field. This ratio of the number of radial and tangential physical scales does not depend on the choice of coordinates or frame of reference, i.e. gravitational contraction of lengths in this sense is absolute.

Clarification of the physical picture in the theory of gravity while restricting the description to hypersurfaces of simultaneity also leads to the solution of problems with gravitational energy and gravitational waves. The hypersurface of simultaneity of the field source's rest frame in these cases represents a physically preferred frame. In this frame, the field energy is determined in the usual way, and the transition to other hypersurfaces is performed by the local Lorentz transformations, so that there will be no problems with field energy. Therefore, in this treatment there are no problems in describing both the energy of the field and the gravitational waves that carry it. These questions will be discussed in more details in subsequent publications.

\subsection{From special relativity to local relativity}

In special relativity (SR), inertial frames in flat space-time are equipped with physical coordinates. The physical distances in them are measured by standard scales, and the physical time by standard clocks. Their intervals form an invariant space-time interval:

$$
d s^{2}=c^{2} d t^{2}-d \mathbf{r}^{2} .
$$

Relativistic effects between inertial frames arise due to the difference in their hypersurfaces of simultaneity. In accelerated moving frames of reference, similar effects also arise and can be detected by introducing the local inertial frames, comoving them at any moment. 
In general relativity, arbitrary coordinate systems were introduced, where the coordinates simply numerate the space-time points, and their intervals do not have direct physical meaning. In particular, hypersurfaces $x^{0}=$ const . in the general case no longer mark simultaneous events, because of what the Cauchy problem becomes a mathematical abstraction without physical content. The space - time interval has the form of the right-hand side of (22) and is invariant with respect to arbitrary transformations $x^{i^{\prime}}=x^{i} \partial x^{i^{\prime}} / \partial x^{i}$ with coefficients that also have no direct physical meaning.

Nevertheless, it was assumed that in some cases it is possible to construct frames of reference similar to SR, when the coordinates become physical. In static fields this is often done, an example of which is the static frame in a spherical field, where world time is entered, which marks simultaneous events. But even in these fields alternative arbitrary coordinates were formally introduced, and then the non-physical results obtained with their help were treated seriously, as having some physical meaning. This introduced into the foundations of physics confusion and elements of arbitrariness.

The diffusion gravity corrects this situation by clarifying the question of separating from the general relativity formalism its physically meaningful part describing the real gravitational fields and the processes occurring in them.

For the gravitational field itself, as was already discussed in the previous section, admissible extended frames of reference are mainly those in which all local elements are at rest with respect to each other and the physical coordinate system is built on a global hypersurface of simultaneity. If the frame of reference is at rest with respect to the source of the field, then such a system describes the influence of the gravitational field on objects and processes in its pure form. It is free from kinematic distortions introduced into the description by the motion of the frame of reference as a whole or by the relative motion of its elements at different points.

But if there is a physical frame of reference with local elements mutually moving at different points, then using the general relativity formalism it is possible to use it, but only subject to the requirements of physical feasibility and admissibility. This is the simultaneous coexistence of local elements of the frame of reference as a whole. This is possible if their local standard scales and intervals of proper time are projected onto the hypersurfaces of simultaneity of the rest frame of one of the elements selected as the basic one. In fact, an extended reference system is constructed from its local systems on the hypersurfaces of simultaneity of the basic local system, despite the relative motion of these elements.

The coordinate systems of such extended frames of reference remain physical, since they are built from standard scales and equipped with standard clocks. They are only projected onto the hypersurface of simultaneity by the local Lorentz transformations. Therefore, the theory of relativity in such a strictly physical form occupies an intermediate position between special relativity and general relativity, being a «localized» form of special relativity or a reduced form of general relativity. Moreover, it is localized not on the arbitrary hypersurfaces, but on a hypersurface of simultaneity. Therefore, this theory could be called as the local theory of relativity, or local relativity.

\section{Conclusion}

Thus, the corresponding thermal diffusion in the background field under certain conditions turned out to be a fundamental consequence of conservative diffusion. This thermal diffusion turned out to be the sought-after physical mechanism of gravity. This circumstance makes the theory of gravity a part of quantum theory and thereby solves the problem of the synthesis of theories of these two phenomena. 
Since this treatment is based on quantum concepts, it implements a new form of synthesis of the theory of gravity and quantum theory. Of the two basic hypotheses of modern physics - quantum fluctuations and gravity - DQM leaves only the first as a hypothesis, and the second is its consequence, i.e. gravity theory becomes part of quantum theory.

The tasks for further research are mainly the causes and mechanisms of fluctuations of the background field, while the adoption of these fluctuations as an observational fact leads to a simple and clear physical picture of both quantum and gravitational phenomena, greatly simplifying the situation in the foundations of physics.

\section{Appendix}

\section{Conservative thermal diffusion of cold light gas in warm heavy}

Diffusion of a light gas in a heavy gas is usually studied at local thermal equilibrium of both components of the mixture, when the mean kinetic energies of both types of atoms are almost equal and therefore friction plays a significant role (Lorentz gas). In this case, the light gas undergoes ordinary diffusion and usual thermal diffusion depending on the concentration distribution and the local temperature of the heavy gas. The diffusion flow $\mathbf{i}$ at a concentration $c$ and thermal diffusion coefficient $D k_{T}$ is given by the expression [5]:

$$
\mathbf{i}=-N D\left(\nabla c+k_{T} \nabla T / T\right) .
$$

The rms velocities of light and heavy atoms are inversely proportional to the mass ratio:

$$
\frac{v_{1}^{2}(x)}{v_{2}^{2}(x)} \sim \frac{m_{2}}{m_{1}}
$$

where $v_{1}(x), v_{2}(x)$, are the local thermal velocities of atoms, $m_{1}, m_{2}$ are their masses. At $m_{1} \ll m_{2}$ heavy atoms of mass $m_{2}$ can be considered as resting (Lorentz gas). As a result, even a small gradient of thermal velocities of heavy atoms of the medium leads to a large gradient of thermal velocities of light impurity atoms.

This leads to stronger thermal diffusion of light particles directed to a colder region. But an increase in the concentration of light particles in the cold region increases the usual diffusion, directed back to the warmer side. The equilibrium condition for these two opposite flows is the disappearance of the total flow $\mathbf{i}=0$ and from (29) we find:

$$
\nabla c=-k_{T} \nabla T / T \text {. }
$$

In the ideal gas approximation, such an equilibrium of two flows is established when the concentration of light gas in a warmer region is higher than in a colder one.

Thus, in the Lorentz gas with local equilibrium diffusion, a light gas, which is more mobile due to the much higher atomic velocity, accumulates in the warmer region. In practice, this property is often used in the separation of gases in mixtures and even isotopes, where the mass difference is small.

For conservative diffusion and thermal diffusion to occur, light gas should be much colder than heavy $T_{1} \ll T_{2}$. This change in conditions leads to the fact that thermal diffusion begins to dominate in such a non-Lorentzian gas and the opposite effect occurs - a light gas concentrates in the colder region [2].

In conservative diffusion, the thermal velocity of light particles is of the same order as the thermal velocity of heavy particles, and the ratio of rms velocities, in contrast to the thermal equilibrium case (30), is much less than the inverse mass ratio: 


$$
\frac{v_{1}^{2}(x)}{v_{2}^{2}(x)} \ll \frac{m_{2}}{m_{1}} .
$$

Since the diffusion coefficient in equilibrium is proportional to the rms velocity of light particles $D \sim T_{1} \sim v_{1}^{2}$, the diffusion flux is significantly weakened with respect to the equilibrium case. At the same time, the thermal diffusion flow of the light particles into the cold region either remains the same or even intensifies, since heavy particles carry light particles into the cold region. As a result of such a violation of the former equilibrium in favor of thermal diffusion flow, it becomes dominant and light gas accumulates in the cold region.

As with ordinary Brownian motion, with random walks of one particle, instead of concentration $n(x, t)$, we are dealing with a probability density $\rho(x, t)$ in an ensemble, and the diffusion flux $\rho \mathbf{u}_{\rho}$ now concerns the probability densities:

$$
\mathbf{i}=\rho \mathbf{u}=-D\left(\nabla \rho+\bar{k}_{T} \nabla T / T\right),
$$

where $D \bar{k}_{T}$ is the corresponding coefficient of thermal diffusion.

In conservative diffusion, there appears thermal diffusion acceleration in addition to the conventional thermal diffusion flow $\rho \mathbf{u}_{T}$. For example, at $\nabla T=$ const. increments of velocity are constant and they accumulate due to a small friction, which means that there appears a constant acceleration $\mathbf{w}=$ const. In this case, the thermal diffusion acceleration, like the flow, is directed to the cold region. The presence of acceleration to the cold region is the second peculiarity of the conservative thermal diffusion.

The full flow thus takes the form:

$$
\rho \cdot\left(\mathbf{u}+\overline{\mathbf{u}}_{T}\right)=\rho \cdot\left(\mathbf{u}_{\rho}+\mathbf{u}_{T}+\mathbf{w} t\right) .
$$

In this case, the diffusion flux $\rho \mathbf{u}_{\rho}=-D \nabla \rho$ is weaker than the thermal diffusion flux $\rho \mathbf{u}_{T}=-D \bar{k}_{T} \nabla T / T$, where the velocity $\overline{\mathbf{u}}_{T}$ increases due to acceleration $\overline{\mathbf{u}}_{T}=\mathbf{u}_{T}+\mathbf{w} t$. This acceleration increases the flow of light particles into the cold region and further enhances the dominance of thermal diffusion in comparison with diffusion directed backward.

Thus, if, under the local thermal equilibrium of the two components, the light gas accumulates in the warm region, then under conservative diffusion, when the light gas is much colder than the heavy, the thermal diffusion flow dominates and the light gas accumulates in the cold region. The thermal diffusion flow also contains acceleration, which in the first approximation does not depend on the light particle's mass.

\section{Maxwell: "Note on the effect of gravity"}

The following is an excerpt from the book of J. C. Maxwell "A Dynamical Theory of the Electromagnetic Field" [4] with his hypothesis about the physical mechanism of gravity.

"After tracing to the action of the surrounding medium both the magnetic and the electric attractions and repulsions, and finding them to depend on the inverse square of the distance, we are naturally led to inquire whether the attraction of gravitation, which follows the same law of the distance, is not also traceable to the action of a surrounding medium.

Gravitation differs from magnetism and electricity in this; that the bodies concerned are all of the same kind, instead of being of opposite signs, like magnetic poles and electrified bodies, and that the force between these bodies is an attraction and not a repulsion, as is the case between like electric and magnetic bodies. 
The lines of gravitating force near two dense bodies are exactly of the same form as the lines of magnetic force near two poles of the same name; but whereas the poles are repelled, the bodies are attracted. Let $E$ be the intrinsic energy of the field surrounding two gravitating bodies $M_{1}, M_{2} \ldots$

If $R$ be the resultant gravitating force, ... Hence

$$
E=C-\sum \frac{1}{8 \pi} R^{2} d V
$$

The intrinsic energy of the field of gravitation must therefore be less wherever there is a resultant gravitating force.

As energy is essentially positive, it is impossible for any part of space to have negative intrinsic energy. Hence those parts of space in which there is no resultant force, such as the points of equilibrium in the space between the different bodies of a system, and within the substance of each body, must have an intrinsic energy per unit of volume greater than

$$
\frac{1}{8 \pi} R^{2}
$$

where $R$ is the greatest possible value of the intensity of gravitating force in any part of the universe.

The assumption, therefore, that gravitation arises from the action of the surrounding medium in the way pointed out, leads to the conclusion that every part of this medium possesses, when undisturbed, an enormous intrinsic energy, and that the presence of dense bodies influences the medium so as to diminish this energy wherever there is a resultant attraction.

As I am unable to understand in what way a medium can possess such properties, I cannot go any further in this direction in searching for the cause of gravitation."

\section{References}

1. Zakir, Z. (2020) Quant. and Grav. Phys., 1:003-7129.

2. Zakir, Z. (2014) TPAC, 9, 19; 9, 34.

3. Zakir, Z. (2020) Diffusion Quantum Mechanics and Diffusion Gravity. CTPA. T.

4. Maxwell, J. K. (1996) A Dynamical Theory of the Electromag. Field, p. 76-77, W.S.P.

5. Lifshitz E. M., Pitaevskii L.P. (1981) Physical Kinetics. Perg. P

6. Zakir, Z. (2016) TPAC, 11, 1.

7. Zakir, Z. (2020) Quant. and Grav. Phys., 1:001-7128.

8. Zakir, Z. (2020) Quant. and Grav. Phys., 1:006-7130; 1:007-7130.

9. Zakir, Z. (2020) Quant. and Grav. Phys., 1:005-7129. 